\title{
ON ISOMORPHISMS BETWEEN CERTAIN NON-CSL ALGEBRAS
}

\author{
MANOS PAPADAKIS
}

(Communicated by Palle E. T. Jorgensen)

\begin{abstract}
In this paper we characterize the existence of algebraic isomorphisms between certain reflexive algebras with two atom lattices. We also show by an example that these isomorphisms need not preserve certain geometric properties of their lattices.
\end{abstract}

\section{INTRODUCTION}

Algebraic isomorphisms between Banach algebras $\mathscr{A}$ and $\mathscr{B}$ of bounded operators on a complex Hilbert space have been investigated by several authors. We study the problem of the existence of isomorphisms between algebras of operators leaving invariant the subspaces of atomic Boolean lattices with precisely two atoms. This is the simplest instance of a nontrivial reflexive algebra whose lattice is not commutative. In our main theorem we give a necessary and sufficient condition for the existence of isomorphisms between reflexive algebras with such lattices. We also prove (Proposition 1.1) that certain geometric properties are not isomorphic invariants.

Whenever $\mathscr{A}$ and $\mathscr{B}$ are operator algebras determined by lattices of subspaces of the underlying space, one basic question is: under what conditions an isomorphism $\phi$ is spatial, i.e.,

$$
\phi(A)=T A T^{-1} \quad \text { for every } A \in \mathscr{A},
$$

where $T$ is a bounded invertible operator. When $T$ fails to be bounded and the previous equation is valid on an appropriate dense subspace, we say that $\phi$ is quasi-spatial (for the precise definition see below).

Notice that when any two operator algebras are similar, they are automatically isomorphic through this similarity. The converse implication fails in general and is of course connected with the problem of spatiality of isomorphisms.

When the lattices in question are totally ordered, every isomorphism between the corresponding algebras, called nest algebras, is spatial, as Ringrose proved in [R]. Therefore, any two isomorphic nest algebras are similar. Our last result investigates to what extent this situation prevails for the algebras under consideration in this paper. Totally ordered subspace lattices form a subclass of

Received by the editors April 1, 1991 and, in revised form, March 25, 1992.

1991 Mathematics Subject Classification. Primary 47D25.

Key words and phrases. Operator algebras, isomorphisms. 
commutative subspace lattices, called CSL, and in addition satisfy a strong condition called complete distributivity. Gilfeather and Moore showed in [GM] that for reflexive algebras whose invariant subspace lattice is CSL and completely distributive, quasi-spatial isomorphisms are those which preserve the rank of finite rank operators. On the other hand, when the lattices are atomic Boolean (hence completely distributive but not necessarily CSL), Lambrou, in [L], proved that all isomorphisms are quasi-spatial.

We mention that many results valid in CSL algebras have been proved for the case of reflexive algebras whose invariant subspace lattice is Boolean with exactly two atoms and some of them have interesting generalizations. One can refer to [AKL, DH, KLL, LL, P].

\section{PRELIMINARIES}

Let $H$ denote a complex Hilbert space and $\mathscr{B}(H)$ the algebra of all bounded linear operators on $H$. All subspaces of $H$ are assumed to be closed. Whenever $\mathscr{L}$ is a complete lattice of subspaces of $H$, we define

$$
\operatorname{Alg} \mathscr{L}=\{A \in \mathscr{B}(H): A(L) \subseteq L \text {, for every } L \in \mathscr{L}\} \text {. }
$$

Clearly $\operatorname{Alg} \mathscr{L}$ is a subalgebra of $\mathscr{B}(H)$, closed, under all relevant topologies.

In [H] Halmos defines two subspaces $M, N$ to be in generic position if $M \cap N=M \cap N^{\perp}=M^{\perp} \cap N=M^{\perp} \cap N^{\perp}=\{0\}$. He also gives the following elegant characterization of generic positioning. We denote by $G(B)$ the graph of the linear operator $B$.

Theorem [H]. If two subspaces $M, N$ are in generic position, there are a Hilbert space $K$ and a positive injective contraction $B$ on $K$ with $I-B$ also injective, such that the pair $\{M, N\}$ is unitarily equivalent to $\{G(B), G(-B)\}$.

The theorem in fact holds with the weaker hypothesis

$$
M \cap N=M^{\perp} \cap N^{\perp}=\{0\} \quad \text { and } \quad \operatorname{dim}\left(M \cap N^{\perp}\right)=\operatorname{dim}\left(M^{\perp} \cap N\right),
$$

but $I-B$ may no longer be injective. We will call this weaker property the generalised generic position.

The join $M \vee N$ of two subspaces $M, N$ is the closure of their linear span in the norm topology of $H$. We call the subspaces $M, N$ quasi-complementary if

$$
M \cap N=\{0\} \quad \text { and } \quad M \vee N=H .
$$

The study of a simple lattice determined by two nontrivial quasi-complementary subspaces is usually reduced to the case of generic position (see [P]). These lattices are complete atomic Boolean whose atoms are the two nontrivial quasicomplementary subspaces.

Now let $\mathscr{A}$ and $\mathscr{B}$ be subalgebras of $\mathscr{B}(H)$. A linear multiplicative map $\phi$ of $\mathscr{A}$ onto $\mathscr{B}$ is called an algebraic homomorphism (or simply homomorphism). If $\phi$ is also bijective, it is called an isomorphism. The isomorphism $\phi$ is said to be quasi-spatial if there is an injective linear transformation $T$ with dense $\mathscr{A}$-invariant domain $D$ and dense range such that

$$
\phi(A) T x=T A x \quad \text { for every } x \in D, A \in \mathscr{A} \text {. }
$$

The rank one operator $e \otimes f$ is defined by the formula

$$
e \otimes f(x)=\langle x, e\rangle f \quad \text { for each } x \in H .
$$


We shall define, now, when two bounded operators are equivalent.

Definition. Two bounded operators $B, D$ have equivalent ranges if and only if there is a unitary operator which maps the range of $B$ onto the range of $D$. In this case we, simply, call $B, D$ equivalent.

In our main theorem, we will need the following characterization of equivalent positive operators.

Theorem. Suppose B, D are two positive operators. Then the following are equivalent.

(i) The operators $B, D$ are equivalent.

(ii) There exist $X, Y$ bounded and invertible operators such that $D=$ $X B Y$.

(iii) There exists a bounded and invertible operator $S$ such that $D=S^{*} B S$.

For details concerning equivalent operators refer to [FW, §3].

\section{GEOMETRIC INVARIANTS OF ISOMORPHISMS}

Isomorphisms between algebras preserve the algebraic structure. Given an isomorphism between $\operatorname{Alg} \mathscr{L}_{1}$ and $\operatorname{Alg} \mathscr{L}_{2}$, where $\mathscr{L}_{1}$ and $\mathscr{L}_{2}$ are two complete subspace lattices, one might wonder if there are any geometric properties of $\mathscr{L}_{1}$ and $\mathscr{L}_{2}$ which remain invariant. Unfortunately, as we shall see in Proposition 1.1 , isomorphisms between reflexive algebras need not preserve some natural geometric properties of their lattices.

1.1. Proposition. Algebraic isomorphisms between reflexive operator algebras need not preserve generalised generic position of pairs of subspaces in their lattices. Proof. Take $K$ to be a separable infinite-dimensional Hilbert space with an orthonormal basis $\left\{e_{n}: n \in \mathbb{N}\right\}$. Take $B$ to be a positive injective contraction on $K$ with $I-B$ also injective. Take

$\mathscr{L}_{1}=\{0, \mathbb{C} \oplus G(I / 2) \oplus G(B), \quad 0 \oplus G(-I / 2) \oplus G(-B), \quad \mathbb{C} \oplus K \oplus K \oplus K \oplus K\}$, $\mathscr{L}_{2}=\{0, G(I / 2) \oplus G(B), G(-I / 2) \oplus G(-B), K \oplus K \oplus K \oplus K\}$.

Since $(I / 2) \oplus B$ is a positive injective contraction with $I \oplus I-(I / 2 \oplus B)$ one to one, the two atoms of $\mathscr{L}_{2}$ are in generic position. It now follows that for $\mathscr{L}_{1}$ we have

$$
[\mathbb{C} \oplus G(I / 2) \oplus G(B)] \cap[0 \oplus G(-I / 2) \oplus G(-B)]^{\perp}=\mathbb{C}
$$

and

$$
[\mathbb{C} \oplus G(I / 2) \oplus G(B)]^{\perp} \cap[0 \oplus G(-I / 2) \oplus G(-B)]=\{0\},
$$

so the two nontrivial subspaces of $\mathscr{L}_{1}$ are not even in generalised generic position.

We shall first obtain a similarity $T$ on $\mathbb{C} \oplus K \oplus K$ which maps $\mathbb{C} \oplus G(I / 2)$, $0 \oplus G(-I / 2)$ onto $G(I / 2)$ and $G(-I / 2)$ respectively. Denoting by $e$ the basic vector for $\mathbb{C}$, define $T$ by

$$
T(e)=\left(e_{1}, e_{1} / 2\right), \quad T\left(e_{n}, e_{n} / 2\right)=\left(e_{n+1}, e_{n+1} / 2\right) \quad \text { for } n \geq 1
$$

and

$$
T\left(e_{n},-e_{n} / 2\right)=\left(e_{n},-e_{n} / 2\right) \quad \text { for all } n \in \mathbb{N}
$$


Then the invertible operator $T \oplus I \oplus I$ on $(\mathbb{C} \oplus K \oplus K) \oplus K \oplus K$ yields a spatial isomorphism between $\operatorname{Alg} \mathscr{L}_{1}$ and $\operatorname{Alg} \mathscr{L}_{2}$.

Remark. Note that the isomorphism constructed in the proof of the previous theorem is spatial; therefore, we can see that even spatial algebraic isomorphisms need not preserve generalised generic position.

\section{MAIN THEOREM}

Before proceeding to our main theorem we must mention some preliminary results about isomorphisms. In [L] Lambrou proved that every algebraic isomorphism between two ABSL algebras (ABSL abbreviates complete atomic Boolean subspace lattice) is always quasi-spatial. One crucial fact used in his proof, adapted for our case, is the following:

2.1. Lemma. Suppose that $\mathscr{L}_{i}=\left\{0, M_{i}, N_{i}, H\right\} \quad(i=1,2)$ with $M_{i}, N_{i}$ nontrivial quasi-complementary subspaces of $H$ and that $\phi$ is an isomorphism between $\operatorname{Alg} \mathscr{L}_{1}$ and $\operatorname{Alg} \mathscr{L}_{2}$. Then there are injective linear transformations (not necessarily bounded) $T$ from $M_{1}+N_{1}$ onto $M_{2}+N_{2}$ and $S$ from $M_{1}^{\perp}+N_{1}^{\perp}$ onto $M_{2}^{\perp}+N_{2}^{\perp}$ such that:

(i) $\phi(e \otimes f)=S e \otimes T f$ for all $e \otimes f$ in $\operatorname{Alg} \mathscr{L}_{1}$.

(ii) The transformation $T$ restricted to $M_{1}$ (resp. $N_{1}$ ) is bounded and onto $M_{2}$ (resp. $\left.\mathrm{N}_{2}\right)$ or vice versa.

Also the transformation $S$ restricted on $M_{1}^{\perp}$ (resp. $N_{1}^{\perp}$ ) is also bounded and onto $M_{2}^{\perp}$ (resp. $\left.N_{2}^{\perp}\right)$ or vice versa.

(iii) $\langle T f, S e\rangle=\langle f, e\rangle$ for all $f \in M_{1}+N_{1}, e \in M_{1}^{\perp}+N_{1}^{\perp}$.

Proof. In Theorem 4.5 of [L] Lambrou constructs a bijective map between the atoms of $\mathscr{L}_{1}$ and $\mathscr{L}_{2}$. The linear transformations $T$ and $S$ are defined in the proof of Theorem 4.8 of [L], with respect to this bijection, while their properties in (ii) of Lemma 2.1 follow from Theorem 4.5 of [L]. Assertion (i) is immediate from [L, relation (3) of the proof of 4.5].

(iii) Choose nonzero $f \in M_{1}$ and $e \in N_{1}^{\perp}$. Then $e \otimes f$ is in $\operatorname{Alg} \mathscr{L}_{1}$. By the multiplicativity of $\phi$ and (i) we have

$$
\phi((e \otimes f)(e \otimes f))=(S e \otimes T f)(S e \otimes T f)
$$

or, equivalently,

$$
\langle f, e\rangle \phi(e \otimes f)=\langle T f, S e\rangle S e \otimes T f
$$

therefore,

$$
\langle T f, S e\rangle=\langle f, e\rangle \text {. }
$$

Similarly we obtain the same equation for $f \in N_{1}$ and $e \in M_{1}^{\perp}$. Since $S, T$ are linear transformations, (iii) follows.

If we now take $M_{1}+N_{1}=M_{2}+N_{2}=H$, it is well known that both lattices are similar to CSL's. Hence it is easy, in this case, to see that $\operatorname{Alg} \mathscr{L}_{1}$ and $\operatorname{Alg} \mathscr{L}_{2}$ are isomorphic if and only if they are similar and that this occurs precisely when $\operatorname{dim} M_{1}=\operatorname{dim} M_{2}$ and $\operatorname{dim} N_{1}=\operatorname{dim} N_{2}$ (or $\operatorname{dim} M_{1}=\operatorname{dim} N_{2}$ and $\operatorname{dim} N_{1}=\operatorname{dim} M_{2}$ ). Therefore, in this case all isomorphisms are spatial. We must notice that the property that $M_{i}+N_{i}$ be closed is equivalent to the angle between $M_{i}$ and $N_{i}$ being nonzero $(i=1,2)$ (see also [P]). 
Consider now the case where $M_{1}+N_{1}=H$ but $M_{2}+N_{2}$ is nonclosed. In this case there is no isomorphism between $\operatorname{Alg} \mathscr{L}_{1}$ and $\operatorname{Alg} \mathscr{L}_{2}$. Indeed, let $\phi$ be an isomorphism of $\operatorname{Alg} \mathscr{L}_{1}$ onto $\operatorname{Alg} \mathscr{L}_{2}$. If we call $P$ the projection on $M_{1}$ along $N_{1}$, then $P$ is bounded because $M_{1}+N_{1}$ is closed, so it belongs to $\operatorname{Alg} \mathscr{L}_{1}$. Hence $\phi(P)$ is an idempotent of $\operatorname{Alg} \mathscr{L}_{2}$. If we let $L$ be the range of $\phi(P)$, then $L$ is a closed subspace, and for each $A \in \operatorname{Alg} \mathscr{L}_{2}$ there is a $D \in \operatorname{Alg} \mathscr{L}_{1}$ such that $\phi(D)=A$. Since $D P=P D P$, for each $x \in L$, we obtain

$$
A x=A \phi(P) x=\phi(D P) x=\phi(P D P) x=\phi(P) A x,
$$

so $A x$ is in $L$. Thus $L$ is invariant under all $A$ in $\operatorname{Alg} \mathscr{L}_{2}$ by the reflexivity of $\mathscr{L}_{2} ; L$ belongs to $\mathscr{L}_{2}$. Similarly $\operatorname{ker} \phi(P)$, which is the range of $I-\phi(P)$, must be $\mathscr{L}_{2}$. Therefore, $L$ must be one of $M_{2}$ or $N_{2}$, while $\operatorname{ker} \phi(P)$ must be the other; hence, $M_{2}+N_{2}$ is closed.

From the above arguments we conclude:

2.2. Proposition. Let $\mathscr{L}_{1}, \mathscr{L}_{2}$ be as in the previous lemma. There exists no isomorphism between $\operatorname{Alg} \mathscr{L}_{1}$ and $\operatorname{Alg} \mathscr{L}_{2}$ if $M_{1}+N_{1}$ is closed while $M_{2}+N_{2}$ is nonclosed. In the case where both $M_{i}+N_{i}(i=1,2)$ are closed, every isomorphism is spatial.

The problem that arises naturally is what happens when both $M_{i}+N_{i} \quad(i=$ $1,2)$ are nonclosed. We answer this problem when $M_{i}, N_{i}(i=1,2)$ are in generalised generic position; however, one can see that, in view of Proposition 1.1 , this is not the only case that can occur.

We can now state our main theorem.

2.3. Theorem. Let $H$ be a complex Hilbert space and $B, D$ two positive injective contractions on $H$. Take

$$
\mathscr{L}_{1}=\{0, G(B), G(-B), H \oplus H\}, \quad \mathscr{L}_{2}=\{0, G(D), G(-D), H \oplus H\} .
$$

If $\phi$ is an algebraic isomorphism of $\operatorname{Alg} \mathscr{L}_{1}$ onto $\operatorname{Alg} \mathscr{L}_{2}$, then $B, D$ are equivalent.

Proof. By Lemma 2.1 there is a linear transformation $T$ such that $T(G(B))=$ $G(D), T(G(-B))=G(-D)$, or vice versa. We may assume that $T(G(B))=$ $G(D)$ by composing $\phi$, if necessary, with the spatial automorphism of $\operatorname{Alg} \mathscr{L}_{2}$ given by $I \oplus(-I)$, which interchanges $G(D), G(-D)$.

By Lemma 2.1, $T$ is a well-defined linear transformation of $G(B)+G(-B)$ onto $G(D)+G(-D)$, which is not necessarily bounded.

For every $x \in H$ there are $v, w \in H$, such that

$$
T(x, B x)=(v, D v) \text { and } T(x,-B x)=(w,-D w),
$$

which yield

$$
\begin{aligned}
& T(x, 0)=\left(\frac{v+w}{2}, D\left(\frac{v-w}{2}\right)\right), \\
& T(0, B x)=\left(\frac{v-w}{2}, D\left(\frac{v+w}{2}\right)\right) .
\end{aligned}
$$

Now define $P(x)=(v+w) / 2$ and $Q(x)=(v-w) / 2$ for all $x \in H$. Now $P$, $Q$ are well defined linear transformations (not necessarily bounded) since $B$, $D$ are injective and $T$ a well-defined linear transformation on $G(B)+G(-B)$. 
In view of equations (1), for all $x \in H$, we have

$$
T(x, 0)=(P x, D Q x)
$$

and for each $y$ in the range of $B$

$$
T(0, y)=\left(Q B^{-1} y, D P B^{-1} y\right) .
$$

Therefore, we can write $T$ in a $2 \times 2$ matrix form relative to the decomposition $H \oplus H$ as

$$
T=\left(\begin{array}{cc}
P & Q B^{-1} \\
D Q & D P B^{-1}
\end{array}\right)
$$

It follows that

$$
T(x, B x)=((P+Q) x, D(P+Q) x) \quad \text { for all } x \in H .
$$

The operator that maps an arbitrary $x \in H$ to the pair $(x, B x)$ is a continuous linear bijection; now $T$ maps $(x, B x)$ to $((P+Q) x, D(P+Q) x)$, and, by taking the projection of $H \oplus H$ onto its first coordinate, we obtain $P+Q$ as the composition of the previously mentioned three linear operators. Since $T$ is a bicontinuous linear mapping on $G(B)$, by (ii) of Lemma 2.1, and $B$, $D$ are injective and bounded, we get that $P+Q$ is a bounded linear bijection on $H$. By a similar argument $P+Q$ must also be invertible.

Following the same procedure we obtain a $2 \times 2$ matrix form for the corresponding operator $S$ of Lemma 2.1 relative to the decomposition $H \oplus H$. This matrix form is

$$
\left(\begin{array}{cc}
D V B^{-1} & D Z \\
Z B^{-1} & V
\end{array}\right)
$$

where $Z, V$ are everywhere defined, not necessarily bounded, linear transformations.

Once again the restriction of $S$ on $G(B)^{\perp}$ is an invertible linear operator onto $G(D)^{\perp}$, by Lemma 2.1(iii). By the same argument as above, one shows that $V+Z$ is a bounded invertible operator on $H$. Lemma 2.1(iii) now yields

$$
\langle T(x, B x), S(B y, y)\rangle=2\langle x, B y\rangle \quad \text { for all } x, y \in H
$$

since one can easily see that $(B y, y) \in G(-B)^{\perp}$. But for all $x, y \in H$

$$
\begin{aligned}
& \langle T(x, B x), S(B y, y)\rangle \\
& \quad=\langle((P+Q) x, D(P+Q) x),(D(V+Z) y,(V+Z) y)\rangle \\
& \quad=2\langle(P+Q) x, D(V+Z) y\rangle .
\end{aligned}
$$

Since for every $x, y$ in $H$

$$
\langle(P+Q) x, D(V+Z) y\rangle=\langle x, B y\rangle,
$$

we finally get

$$
B=(P+Q)^{*} D(V+Z) .
$$

The operators $P+Q$ and $V+Z$ are bounded and invertible; therefore, the last equation shows that $B$ and $D$ are equivalent.

The question that naturally arises is if the converse implication holds. Our next theorem answers this question. 
2.4. Theorem. Let $\mathscr{L}_{i}(i=1,2)$ be as in the previous theorem. Suppose that $B, D$ are equivalent. Then there exists a spatial isomorphism $\phi$ between $\operatorname{Alg} \mathscr{L}_{1}$ and $\operatorname{Alg} \mathscr{L}_{2}$.

Proof. One can easily verify that $\operatorname{Alg} \mathscr{L}_{1}$ consists of all matrices of the form

$$
\left(\begin{array}{cc}
X & Z \\
B Z B & Y
\end{array}\right)
$$

with $Z$ arbitrary and $X, Y$ satisfying $B X=Y B$, while the operators in $\operatorname{Alg} \mathscr{L}_{2}$ have the same matrix form with $D$ replacing $B$.

As mentioned in the preliminaries about equivalent operators, there is a bounded and invertible operator $S$ such that

$$
D=S^{*} B S \text {. }
$$

We define $\phi$ by

$$
\phi(A)=T A T^{-1} \quad \text { for all } A \text { in } \operatorname{Alg} \mathscr{L}_{1},
$$

where $T=S^{-1} \oplus S^{*}$. Using relation (2) and the above matrix forms for the operators in $\operatorname{Alg} \mathscr{L}_{i} \quad(i=1,2)$, it can easily be verified that $\phi$ is well defined and onto. The definition of $\phi$ shows that it is an algebraic isomorphism and this completes the proof.

As mentioned in the introduction, Ringrose proved that isomorphic nest algebras are exactly those which are similar. A combination of Theorems 2.3 and 2.4 yields the same result for algebras leaving invariant two atom Boolean lattices in generalised generic position:

2.5. Theorem. Let $\mathscr{L}_{1}, \mathscr{L}_{2}$ be atomic Boolean lattices with two atoms in generalised generic position. Then $\operatorname{Alg} \mathscr{L}_{1}$ and $\operatorname{Alg} \mathscr{L}_{2}$ are algebraically isomorphic if and only if they are similar.

Ringrose proves similarity of isomorphic nest algebras by showing that any isomorphism must be spatial. Our proof is indirect: Given an isomorphism we define another, perhaps different, which is spatial by construction. Thus the following questions naturally arise:

(1) If $\mathscr{L}_{1}, \mathscr{L}_{2}$ are atomic Boolean lattices with two atoms in generalised generic position, are all algebraic isomorphisms between $\operatorname{Alg} \mathscr{L}_{1}$ and $\operatorname{Alg} \mathscr{L}_{2}$ spatial? If not, can we find sufficient conditions for spatiality?

(2) What happens if we drop the assumption of generalised generic position? Can we still have the conclusion of Theorem 2.5?

Added in Proof. Recently an example of a nonspatial automorphism of a reflexive algebra of operators on a Hilbert space, leaving invariant a complete atomic Boolean lattice with countably many one-dimensional atoms, was constructed in [KLP]. The first of the above questions has been affirmatively answered in the case of separable Hilbert spaces (see [LAL]).

\section{ACKNOWLEDGMENT}

I must express my thanks to Professors Katavolos and Lambrou for many enlightening conversations and the referee for his useful suggestions during the preparation of the present paper. 


\section{REFERENCES}

[AKL] M. Anousis, A. Katavolos, and M. S. Lambrou, On the reflexive algebra with two invariant subspaces, J. Operator Theory (to appear).

[DH] K. Davidson and K. Harisson, Distance formulae for subspace lattices, J. London Math. Soc. 39 (1989), 309-323.

[FW] P. A. Fillmore and J. P. Williams, On operator ranges, Adv. in Math. 7 (1971), 254-281.

[GM] F. Gilfeather and R. Moore, Isomorphisms of Certain CSL algebras, J. Funct. Anal. 67 (1986), 264-291.

[H] P. Halmos, Two subspaces, Trans. Amer. Math. Soc. 144 (1969), 381-389.

[KLL] A. Katavolos, M. S. Lambrou, and W. E. Longstaff, The decomposability of operators relative to two subspaces, Studia Math. (to appear).

[KLP] A. Katavolos, M. S. Lambrou, and M. Papadakis, On some algebras diagonalised by $M$ bases of $l^{2}, \mathrm{~J}$. Integral Equations Operator Theory (to appear).

[L] M. S. Lambrou, Automatic continuity and implementation of homomorphisms, preprint.

[LAL] M. S. Lambrou and W. E. Longstaff, Spatiality of isomorphisms between certain reflexive algebras, preprint, 1993.

[LL] - Unit ball density and the operator equation $A X=B Y$, J. Operator Theory 25 (1991), 383-397.

[P] M. Papadakis, On hyperreflexivity and rank one density for non-CSL algebras, Studia Math. 98 (1991).

[R] J. Ringrose, On some algebras of operators. II, Proc. London Math. Soc. 3 (1966), 385-402.

\section{GREECE}

Department of Mathematics, University of Athens, Panepistimiopolis, 15784 Athens,

Current address: 93 Abidou Str., 15771 Zografou, Hellas, Greece

E-mail address: manos papegrathun1.bitnet 